\title{
Wireless Communication in Process Automation: A Survey of Opportunities, Requirements, Concerns and Challenges
}

\author{
Waqas Ikram \& Nina F. Thornhill \\ Centre for Process Systems Engineering, Imperial College London, London SW7 2AZ, UK \\ (e-mail: waqas.ikram08@imperial.ac.uk,n.thornhill@imperial.ac.uk).
}

\begin{abstract}
The advancements in wireless networking technology, specifically in the short-range wireless networking technology, offer an enormous opportunity for wireless connectivity of field devices both in oil and gas and other chemical processing plants. The prerequisite of a field network includes real-time support for mixed traffic, availability, security, reliability and scalability in a harsh industrial environment. These conditions have to be fulfilled by any wireless network in order to operate. This paper presents a brief overview of the requirements for wireless in process automation, relative standings of existing short-range wireless network technologies based on the outlined criteria, and associated shortcomings. Furthermore, an examination of emerging industrial wireless standards which are designed to address the unique and stringent requirements of the process industry, is presented.
\end{abstract}

Keywords: Wireless, Process Automation, Real-Time, Fieldbus, WirelessHART, ISA100.11a

\section{INTRODUCTION}

Plant knowledge is essential for the process industry in order to improve process operations, productivity and on-site safety. Such knowledge can be enhanced by acquiring further information from the underlying processes, which leads to increased visibility (Ferris, 2010). Further, knowledge management in effect involves sharing of information which has its own challenges including information acquisition, management, integration and dissemination of this knowledge (Egea-Lopez et al., 2004). A significant part of the solution to these challenges is wireless technology, which holds enormous potential for providing cost-effective and proficient solutions for data acquisition and effective sharing of information across the facility [Jämsä-Jounela (2007), Ferris (2010), Egea-Lopez et al., (2004), Wedge (2010)].

\section{Why Use Wireless?}

Global competition is driving the industry to continuously improve process operations, product quality, productivity, reliability and compliance with regulations (IWC, 2002). Wireless networks can assist the process industry to gather more data from processes, predict maintenance of equipment, increase workforce efficiency through plant-wide network connectivity and provide low-cost connectivity solutions (Emerson 2009). Wireless technology is attractive as it eliminates the problems associated with wired networks.

Problems with wired technology:

- Pre-planning requirements, higher installation and maintenance costs of the wired network (Ferris, 2010).

- Difficulty in troubleshooting connectors (Hartebrodt $e t$ al., 2004).
- Less flexible infrastructure due to fixed connections.

- Wired networks have to be designed with spare capacity on cards, marshalling cabinets, junction boxes and so forth, to cater for future expansion (Ferris, 2010).

- Rotating equipment cause constant twisting of cables which results in fatigue and communication failure.

Key Benefits of wireless technology:

- Wear-and tear free data transfer.

- Lower installation and maintenance costs (Ferris, 2010).

- Deployment opportunities in mobile, and rotating equipment, and hostile and remote locations.

- Deployment opportunity for MEMS (MicroElectromechanical Systems) technology, advantages of which include cost efficiency, low power, high performance sensing and integration (Flammini et al (2008).

- Fairly reliable communication without expensive connectors (Forgue, 2010).

The use of wireless technology can assist the industry to overcome the limitations of wired networks, and benefit from the mobility and design freedom it offers.

The rest of the paper is organised as follows. Section 2 highlights the opportunity for wireless in the process industry and particularly in process automation, followed by network requirements in section 3. Section 4 deals with concerns and challenges regarding adopting wireless in a plant environment. The review of existing short-range wireless network technologies and their standings with respect to these requirements is presented in section 5. The paper concludes with a summary of key aspects of this review in section 6 . 


\section{WIRELESS IN PROCESS INDUSTRY}

The cost-efficient operation of a plant is highly dependent on the automation systems (Drathen, 2009). (Drathen, 2009) reports that the use of wireless technology and the internet will be significant in future process automation solutions. Wireless can help reduce blind spots in process visibility and achieve higher yields and better quality at lower costs (Isaksson, 2009). Some examples of such applications are: Predictive maintenance: Mechanical failures of equipment like motors and drives are amongst the most common causes of production stoppage according to Ralston (2007). Predictive measures using vibration monitoring and data analysis tools for prevention of machine or plant shut-down do exist. However, the cost and feasibility of using wired networks with these systems is sometimes prohibitive. Wireless has a clear advantage in this aspect. Compliance with regulations: The Process Safety Management standard requires an organisation to have compliance audits, workplace process hazard analysis, implement plans to maintain integrity of process equipment and so forth. Wireless enabled devices can help achieve these objectives (William, 2010).

Field rounds: Various tasks in and around process plants are often documented manually by engineers in the field. Their senses like eyes, ears and sense of smell often give indication of process problems, especially if the operator is experienced. This manual process is time consuming, requires man-power and is not efficient. The use of distributed wireless sensor networks can help to collect this data, disseminate this data to concerned authorities, eliminate manual rounds and improve responsiveness to process problems.

Safety: Various applications related to safety are found in industry, such as, safety shower monitoring, fire and gas detection and elimination of spills to avoid safety violations. The use of wireless can help increase safety by having fewer blind spots around the plant leading to enhanced visibility, hence, resulting in improved safety on site.

Harsh environment and rotating equipment: The use of wired communication is limited in certain applications due to either technical or economical reasons (Prosoft, 2010). Access to harsh environments and rotating equipment are some examples. Wireless communication offers an opportunity to access these locations and replace slip-rings and festoon cable (Forgue, 2010).

Management of stranded assets: Management of resources such as workforce and equipment is important to companies. Supply chain optimisation requires integration and automation of the tracking of mobile field operators or equipment in plant (William, 2010).

The use of wireless is not limited to only monitoring applications in a plant environment but can also be extended to control.

\subsection{Wireless in Process Automation}

Process automation uses sensors which are distributed at various locations around the plant to collect the process data, which is further used for various tasks such as calculation of control action, simulation of different operation modes and data archiving. In short, the information collected is used to manage the plant. Automation is becoming increasingly important to process industries such as chemical, oil and gas, and pharmaceutical industries in both industrialised and developing countries (Jamsa-Jounela, 2007). The motivation for applying process automation in industrialised countries is to enhance product quality, improve process safety and availability, and better resource utilisation; mass production is the incentive for developing countries (Jamsa-Jounela, 2007). Wireless is predicted to be one of the fastest growing technologies in the area of process automation sector, and is expected to penetrate into process field devices (ARC, 2008).

Industrial automation systems comprise of various field devices and technologies working in synchronisation. These devices are responsible for a variety of functions related to instrumentation, control, supervision and operational management (Paavola, 2007). The hierarchical structure of the industrial automation systems, based on ISA-95 functional hierarchy is shown in figure 1, also known as the automation pyramid. The hierarchical levels define stages at which decisions are made. The root of automation solutions is in the production process, and goes all the way to enterprise management level via different intermediate layers. The diagram also provides information about the networking requirements at various layers which is the reason why various communication technologies are used. Such distinction occurs in terms of volume of data, timeliness of information, reliability and robustness (Sikora, 2007). Operating conditions on the plant floor are also often harsh compared to the ones present at the higher layers. At the field level, the choices include pneumatic, $4-20 \mathrm{~mA}$ systems, digital fieldbuses and emerging wireless solutions. The two sides of pyramid visible in figure 1 represent the same functional layers. The left face demonstrates the architecture of components based on wired communications technology while the right face shows the wireless domain. This emerging wireless phase in the process automation sector is not just limited to the field level; it can also be used at the plant network level. Figure 2 shows the networking architecture associated with field networks. Wireless can reduce installation costs, equipment costs, time and spare capacity designed for future expansion (Emerson, 2010).

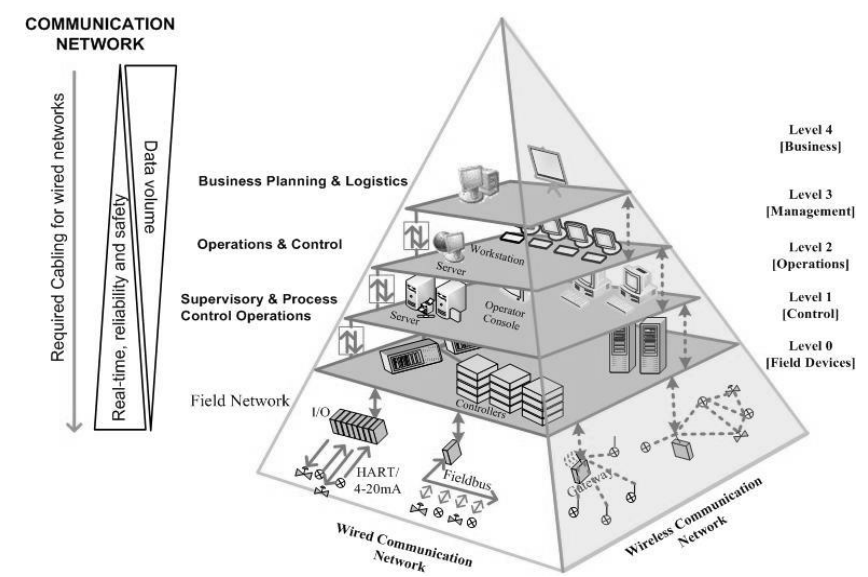

Fig. 1. Automation pyramid based on ISA-95 functional hierarchy. 


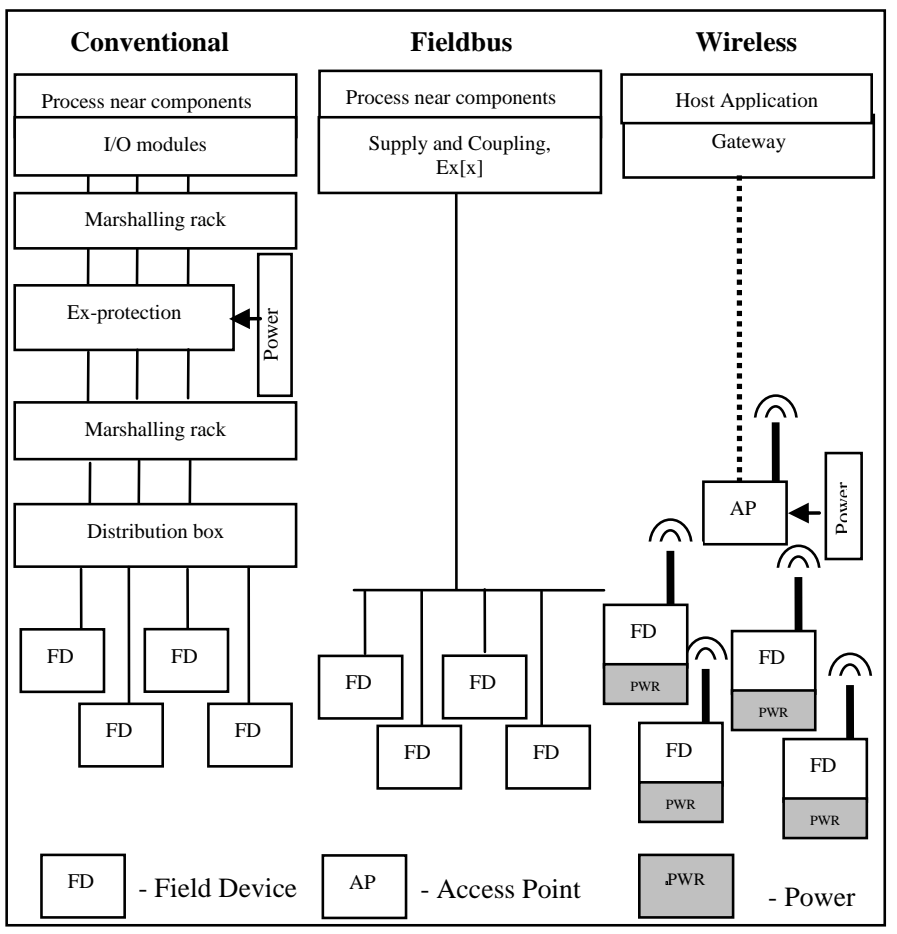

Fig. 2. Comparison between conventional, fieldbus and wireless network architecture. After Kress-Rogers et al (2002) and Ferris (2010).

\section{FIELD NETWORK REQUIREMENTS}

This section examines the networking requirement at the field level. A field bus is the spinal column of real- time distributed systems, providing means for connecting field devices such as sensors, actuators and field controllers (Thomesse, 1998). As the fieldbuses are widely used, any alternative or auxiliary solution to existing fieldbus technology has to meet the performance levels of these systems, and in addition offer improved services.

\section{Basic Requirements:}

Data Handling and Real-time transmission: The network should have the capability to handle and cater for traffic associated with periodic data, sporadic data and network configuration data (messages). Periodic data is mostly linked to inputs and outputs of control algorithms. This information has to be transported within a given time before a new sample is taken (Decotignie, 2002). Sporadic traffic, for example alarm data, has a given bounded latency. The messages are non real-time and are related to network maintenance (Choi et al., 2008).

Order of Events: The orders in which events occur have to be identified for sporadic traffic, and this information is vital for applications that make decisions (Decotignie, 2002).

Availability: The availability of network is very critical, since information flow is vital to control of the physical process or plant.

Safety: Radio waves can themselves cause ignition in a flammable environment if the conditions are favourable and the radio wave transmission levels are high (Schultz, 2007). Therefore, emission levels should be kept under certain levels as specified in the British Standard (BS6656, 2002).

Data Length and Bandwidth: Typical data length is short (Ahmad et al., 2008) and the bandwidth required per node is generally low (Sikora, 2007).

Range: The range between two communicating nodes can be medium to large in size, dependent on the structural layout. Node Size: Compactness is not a main requirement (Flammini et al., 2008).

Scalability: The network has to accommodate multiple nodes, hundreds to even thousands in number (Sikora, 2007). Wireless Associated Requirement:

Mobility: The network should offer connectivity to both static (e.g. fixed equipment) and mobile (e.g. user terminal) nodes in the field.

Security: Information should be encrypted and protected against eavesdropping as it may contain information about the workflow (Sikora, 2007). Additionally, malicious packets should be discarded (Person, 2007).

Duty Cycle: Duty cycle is relatively low per node. If all devices are operated as routers, then duty cycle will increase when the radio traffic accumulates (Sikora, 2007). Energy Consumption: If battery operated, energy consumption should be kept low; battery life of greater than 5 years is preferred (Kang, 2008). Otherwise, a constant need for battery replacement will create a maintenance problem. Reliability: High reliability is a key requirement in process automation. Reliability is a measure of the percentage of transmitted data packets received at the receiver node (Doyle et al., 2008).

Interference: Wireless is an open access medium and therefore, operation in a license free band network will need to be immune to interference. Adaptability to both short-term and long-term interference is vital (Dust Networks, 2009). Delivery: Wireless networks can be affected by noise and interference which can degrade channel performance. Appropriate and timely measures should be adopted to provide data retransmission in case of packet loss.

Other requirements which are not addressed in this paper are related to commissioning, deployment and maintenance of the network.

\section{CONCERNS AND CHALLENGES}

The use of wireless in a plant facility raises concerns regarding the operational hazard of radio waves in the plant and the potential of wireless networks to stand up to the challenges of meeting rigid requirements.

\section{Concerns:}

Radio-waves in Hazardous Environments: Electromagnetic waves can cause currents to flow in metallic structures and if that flow is interrupted momentarily, sparks can occur resulting in ignitions in flammable atmosphere if induced voltage and current is large (Schultz, 2008). The higher the electromagnetic radiation expected in a particular region, the higher the risk to which the site is exposed if dealing with 
flammable fluids. Threshold power required for a particular ignition depends on the gas group. BS6656 contains detailed information related to the gas groups and associated threshold power levels required to ignite them. For example radio power exceeding $6 \mathrm{~W}$ and $2 \mathrm{~W}$ can cause ignition to Propane and Hydrogen respectively in the right conditions for extracting sufficient power from the radio waves. Wireless technology required for wireless process automation involves power levels which are well below the threshold of ignition of all gas groups. Output power from WPAN and WLAN devices is up to $100 \mathrm{~mW}$. Even considering reflections, modulation factors and other safety margins, the power levels in plants due to the presence of onsite WPAN \& WLAN devices will be well below the levels which can cause an ignition hazard. The example given below provides an insight into the expected received power of a radio wave for wireless process automation.

Output power from transmitter $(100 \mathrm{~mW})$

$+20 \mathrm{dBm}$

Transmit antenna gain:

$+5 \mathrm{dBi}$

Coax cable attenuation:

$-3 \mathrm{~dB}$

Basic transmission loss (10m between antennas): $\quad-60 \mathrm{~dB}$

Enhancement factor:

Receiver antenna gain:

$+6 \mathrm{~dB}$

Receiver feeder loss:

$+5 \mathrm{dBi}$

Received power:

$-30 \mathrm{dBm}(\sim 1 \mu \mathrm{W})$

Reliability and Fail-Safe Operation: Radio transmission suffers from bit error rates (BER) which are higher by orders of magnitude compared to cables, where BERs range from $10^{-7}$ to $10^{-9}$ (Decotignie, 2002). This requires the use of error correction techniques. If a communication in a wireless network is unreliable then it can lead to missed input output data (IO). After certain number of failed attempts the loop will be declared failed and shifted to fail-safe mode. Constant occurrence of such events is undesirable (Song et al., 2007).

Frequency Spectrum Jamming: The use of one static channel can be subject to jamming and interference leading to communication degradation. This is one of the reasons why ZigBee was not a viable option for industrial applications, as will be explained later. This issue is being addressed by the industry using spread spectrum techniques like frequency hopping (Caro, 2007).

There are other concerns which are related to energy supply, co-existence in the licence free band, snooping and so forth.

\section{Challenges:}

Amongst the various challenges which are associated with the use of wireless communication, two will be briefly discussed here are related to security and interference.

Data and Network Security: Tapping into cables requires physical access. The use of wireless for transportation of vital process information hence requires incorporation of data encryption and advanced security measures.

Interference: The use of licence free radio channel for communication is susceptible to interference from other nearby sources operating in the same band. If the ISM (Industrial, Scientific and Medical) band is to be used for communication, then it requires network coordinator to continuously assess the channel status to ensure reliable communications.
Other challenges include: guaranteed real-time delivery in unprotected radio spectrum, robustness, power management, effective utilisation of limited bandwidth and so forth.

\section{WIRELESS COMMUNICATION TECHNOLOGIES}

Wireless technologies are often categorised based on the coverage they offer, which ranges from meters to several kilometres. The range requirement is application dependent, for instance the requirements for wireless coverage in factory automation is relatively low compared to process automation. Figure 3 provides an overview of different wireless networks, their associated standards and properties. Figure 3 has three axes: range (distance between communicating devices), transmit power (output power level of transmitter) and channel rate (speed of communication). At an enterprise level, the use of external resources like satellite and fixed telephony can be used for long range communications, whereas within the industrial environment, technologies covering WPAN and WLAN are of wider interest. In these regions the communication range is up to $100 \mathrm{~m}$ and the transmit power allowance is low, but the data rate depends on the technology being used as can be seen in figure 3 .

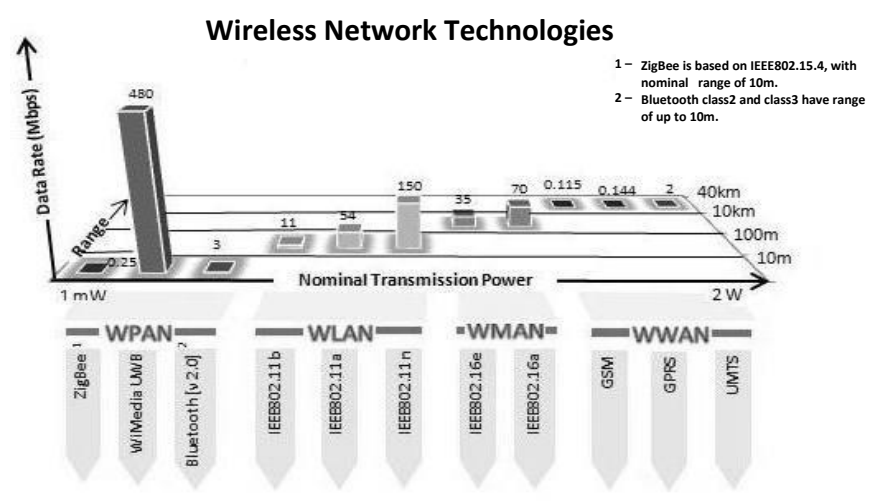

Fig. 3. Illustration of wireless network technologies based on channel rate, transmit power and range.

ZigBee: It is attractive for simple applications with low data rate, low power and low cost requirements. It offers diversity of channel selections over three bands: one in $868 \mathrm{MHz}$ (Europe); ten channels in $902-928 \mathrm{MHz}$ (USA) and sixteen available in the $2.4 \mathrm{GHz}$ ISM band (Worldwide). It has low Quality-of-Service (QoS) guarantee, it is not industrial grade, does not support determinism, and does not employ frequency hopping making it susceptible to interference. Bluetooth: Bluetooth operates in the $2.4 \mathrm{GHz}$ (ISM) band.

Piconet is the basic unit of networking in Bluetooth; it is an ad-hoc network with one master and up to seven active slave nodes (Bluetooth, 2009). Bluetooth uses FHSS to avoid interference by hopping to a new frequency 1600 times a second. Bluetooth is less favourable for process automation due to the complexity of its protocol, scalability problems and lack of flexibility in topology (Vergetis et al., 2004). $U W B$ : Ultra-wideband technology is designed for applications which require a power-efficient, high bandwidth solution. UWB offers enormous bandwidth which makes it eligible to tackle multipath and jamming issues (UWB, 
2009). UWB technology has different frequency band allocation in Europe and USA along with two distinct and incompatible standards known as WiMedia and DS-UWB (Liang et al., 2007). There are still reservations related to the use of UWB technology as it has potential to interfere with many other users due to its wider spectrum.

IEEE 802.11a/b/g: The IEEE 802.11 specification describes the characteristics of a WLAN. Compared to WPAN, they are relatively expensive, devices affiliated with a particular access point share the same bandwidth and the packets are dealt in the order received. Less focus has been paid to factors which are vital to the automation industry such as power, real-time data delivery, QoS, reliability and scalability amongst others (Chen et al., 2004).

Industrial Perspective: Any problem with the equipment or plant translates into economical loss, hence if the equipment is operated wirelessly, the reliability of the network is of utmost importance. Therefore, parameters like robustness, guaranteed message delivery, security and integrity are important for industrial use. According to Backer (2005), ZigBee can meet a wide variety of industrial application needs compared to Bluetooth due to its longer battery life, range (which can be extended through multi-hop) and mesh networking. ZigBee however uses one static channel for communication in a network, which makes it susceptible to interference and jamming. Interference from other sources can cause packet delays and even data loss which makes ZigBee less suitable for industrial control applications (Lennvall et al., 2008). Wi-Fi is also not suitable for industrial applications because of its higher power consumption compared to the others, less secure $802.11 \mathrm{a} / \mathrm{b} / \mathrm{g}$ network and problems reported for operation in electrically noisy environments (Caro, 2009).

Industrial Communication Technologies: There are several standards which have emerged to address these industrial needs are documented below.

Table 1 provides a technical comparison between these industrial protocols.

\begin{tabular}{|l|c|c|c|}
\hline & ZigBee PRO & WirelessHART & ISA 100.11a \\
\hline Transceiver & IEEE 802.15.4 & IEEE 802.15.4 & IEEE 802.15.4 \\
\hline Topology & Mesh, Tree & Mesh & Mesh, Tree \\
\hline Channel hopping & Agility & Hopping & Hopping \\
\hline Encryption & AES128 & AES128 & AES128 \\
\hline $\begin{array}{l}\text { Superframe slot } \\
\text { size }\end{array}$ & N/A & $10 \mathrm{~ms}$ & $(10-12 \mathrm{~ms})$ \\
\hline Channel access & CSMA & TDMA & $\begin{array}{c}\text { TDMA and } \\
\text { contention access }\end{array}$ \\
\hline $\begin{array}{l}\text { Frame Integrity } \\
\text { Check }\end{array}$ & 32 bit & 32 bit & 32-bit or 64-bit \\
\hline $\begin{array}{l}\text { Expansion/ New } \\
\text { Determinism }\end{array}$ & $\begin{array}{c}\text { Extension of } \\
\text { ZigBee }\end{array}$ & $\begin{array}{c}\text { Extension of } \\
\text { HART protocol }\end{array}$ & New protocol \\
\hline $\begin{array}{l}\text { Channel } \\
\text { blacklisting }\end{array}$ & $\begin{array}{c}\text { Preferred } \\
\text { channel }\end{array}$ & Ylacklist & Yes \\
\hline Battery Life & Best & Good & Good \\
\hline $\begin{array}{l}\text { Reliability in harsh } \\
\text { environment }\end{array}$ & Low & Good & Good \\
\hline
\end{tabular}

Table1. Comparison of Industrial Communication Protocols, sources (ISA100.11a, 2009), (WirelessHART, 2008) and (ZigBee, 2010).

WirelessHART: It is a wireless mesh network protocol designed by the HART Communication Foundation for wireless transmission of HART messages. WirelessHART utilises a time synchronised, self-organised, self-healing and redundant path mesh architectural network which operates in the $2.4 \mathrm{GHz}$ ISM band.

ISA100.11a: This is a wireless networking standard developed by ISA for process control and related applications. In general the application focus is to address performance needs for periodic monitoring and control applications where latencies in the order of $100 \mathrm{~ms}$ or above are tolerated (ISA100, 2009). It is standard aimed at multiprotocol capability.

ZigBeePRO: It is an extension of ZigBee which enhanced security features and frequency agility to address the requirements (ZigBee, 2010).

Reliability in the harsh industrial environment and associated security has been widely considered in the formulation of these protocols. Current industrial focus to achieve reliability is by using mesh networks, with packet by packet frequency hopping. Mesh networks however increase latency, which is a concern for control applications and battery operated devices. Data encryption, integrity check and topology are almost similar in all these standards. Due to real-time requirements of the process automation network, Time Division Multiple Access (TDMA) based channel access is important. The duration of channel access (slot size) in WirelessHART is fixed to $10 \mathrm{~ms}$, whereas, in the case of ISA100.11a is configurable to $10-12 \mathrm{~ms}$, giving room for manoeuvre. This makes WirelessHART and ISA100.11a appealing; they are both tailored solutions for process automation applications with a current focus on monitoring applications.

\section{Ongoing Issues:}

Implementation: WirelessHART protocol implementation has its own associated challenges (Song et al., 2008) including: timer design, synchronisation and security. The hardware requirement of network nodes to implement a complete WirelessHART stack also requires higher processing capability and memory. There are also some other features such as effective handover of the network nodes if they are mobile, which have not being addressed in the standard (Kim et al., 2008).

Control application over wireless: The effort so far from industry has concentrated on the use of wireless for monitoring applications and less towards control, which provides an alternative avenue to explore. In a conventional wired communication network for process automation, control commands are executed periodically. This model of periodic execution can be adapted in an alternative wireless communication network but may not be a viable option because of limited bandwidth and onboard power. Innovative solutions like smart publishing and event-triggered control as proposed by Johansson (2009) can address such issues. The use of wireless communication for control is a crossdisciplinary research; it requires an understanding of trade-off analysis between control and wireless networking parameters.

\section{CONCLUSION}

Wireless technology holds enormous potential for the process industry to meet their rising demands while maximising potential at reduced cost with less constrained infrastructure. 
The requirements at field level are stringent compared to higher levels of the automation hierarchy; nevertheless they are achievable. It can be summarised that the short-range and low-power wireless communication technologies are safe for process automation applications. The challenges and concerns regarding interference and jamming are being addressed by industry using schemes like channel hopping and frequency agility. The two new standards, wirelessHART and ISA100.11a are both well positioned to address the industrial needs especially because of the advance security, channel hopping, TDMA and blacklist-based approach to guarantee real-time operations and reliability in an unprotected licence-free radio spectrum. As these two standards are relatively new, few compliant products have so far emerged in the market. Some first generation devices are staring to emerge and it will be interesting to follow developments in the next few years.

\section{Acknowledgments}

The authors would like to acknowledge the contributions from Prof. Geoffrey Maitland of Imperial College, Dr. John Pretlove and Dr. Paula Doyle of ABB for their contributions towards this paper. The authors acknowledge the DTA Scholarship from Imperial College and funding from the ABB/RAEng Chair of Process Automation.

\section{REFERENCES}

ABB (2006), 'Process Automation: ABB Technology Guide', Available at: http://www.abb.com/ [Accessed on 10th February 2010].

Baker, N., "ZigBee and Bluetooth: Strengths and weaknesses for industrial applications", IEEE: Computing and Control Engineering, vol 16, pp 20-25, April/May 2005.

British Standard BS6656:2002, "Assessment of inadvertent ignition of flammable atmospheres by radio-frequency radiation".

Caro, D. May 2009, "Wireless Networking in Plant", retrieved from http://isa.org/InTechTemplate.cfm [Accessed on 14th September 2009]

Chen, D et al. 2004, "Wireless Process Control Products from ISA 2004", Austin USA.

Choi, D. \& Kim, D. (2008), 'Wireless Fieldbus for Networked Control Systems using LR-WPAN', Internation Journal of Control, Automation, and Systems 6, 119-125.

Drathen, H., Process Automation Trends, ACHEMA Worldwide News, Available at: http://www.dechema.de/ dechema_media/. [Accessed on 20th November 2009]

Dust Networks, The benefits of smart dust and mesh technology. Available at:

http://www.dustnetworks.com/multimedia/smartdust_and_mesh/ [Accessed on $10^{\text {th }}$ December 2010]

Egea-Lopez, E.; Martinez-Sala, A.; Vales-Alonso, J.; Garcia-Haro, J. \& Malgosa-Sanahuja, J. (2005), 'Wireless communications deployment in industry: a review of issues, options and technologies', Computers in Industry, 29 - 53.

Emerson 2009, Emerson's Smart Wireless Thum Adapter upgrades existing HART instruments to access

diagnostics and process variables, Available at:http://www.emersonprocess.com. [Accessed on 21st September 2009]

Ferris, M. (2010), 'Next-Gen Wireless in Control Systems. Real World Wireless', 23rd February [Presentation]: IET London: Savoy Place, UK.
Flammini, A et al 2008, "Wired and wireless sensor networks for industrial applications", Elsevier, Microelectronics Journal, Volume 40, Issue 9, Sep 2009, Pages 1322-1336.

Forgue, B. (2010), Next-Gen Wireless in Control Systems, Going Wireless: Why and How?, 23rd February [Presentation]: IET London: Savoy Place, UK.

Hartebrodt, M. \& Kabitzsch, K. (2004), Fault detection in fieldbuses with time domain reflectometry, in AFRICON, 2004. 7th AFRICON Conference in Africa', pp. 391 -396

IWC. (2002), Industrial Wireless Technology for the 21st Century, Available from http://www.energetics.com/ [Accessed on 12th January 2008]

Jämsä-Jounela, S.-L. (2007), 'Future trends in process automation', Annual Reviews in Control 31(2), $211-220$.

Kennedy, J. F. \& Mistry, J. (2003), 'Instrumentation and Sensors for the Food Industry: E. Kress-Rogers, J.B. Brimelow (Eds.); Woodhead Publishing Ltd, Cambridge, UK, 2001Carbohydrate Polymers 51(3), 362 - 363.

Kim, A et al. 2008, "When HART Goes Wireless: Understanding and Implementing the WirelessHART standard", Emerging Technologies and Factory Automation, 2008. ETFA 2008. International Conference on, Sep 2008, Pages 899-907.

Lennvall, T \& Svensson, S 2008, "A Comparison of WirelessHART and ZigBee for Industrial Applications".

Liang, Y et al. 2007, Emerging Wireless Standards for WRAN, WiFi, WiMedia and ZigBee", Infocomm Research.

Paavola, M. (2007), 'Wireless Technologies in Process Automation A Review and an Application Example', Retrieved from http://herkules.oulu.fi/isbn9789514287053.pdf http://herkules.oulu.fi/isbn9789514287053.pdf.

Persson, L. (2007), 'A comparison between fieldbuses and I/O for Instruments in the Process Industry', Available at: http://epubl.luth.se/1402-1617/2007/010/LTU-EX-07010-SE.pdf [Accessed 18 March 2010].

Schultz,S, "Wireless Goes Process Automation - Challenges in Hazardous Areas" Germany.

Sikora, A., Wireless for Industrial and Process Automation-Trends, Challenges, and Protocol", Available at: http://www.baloerrach.de [Accessed on $10^{\text {th }}$ May 2009]

Song, J.; K. Mok, A.; Chen, D. \& Nixon, M. (), 'Challenges of Wireless Control in Process Industry', Available at: http://moss.csc.ncsu.edu/ mueller/crtes06/papers/009-final.pdf.

[Accessed on: 14 March 2009]

Song, J et al (2008)). WirelessHART: Applying Wireless Technology in Real-Time Industrial Process Control.

Thomesse, J. P. (1998), 'A review of the fieldbuses', Annual Reviews in Control 22, 35 - 45.

UWB, "Ultra-Wideband Technology", retrieved from http://www.intel.com/go/uwb/ on $16^{\text {th }}$ August 2009.

Vergetis, E et al. 2004, Can Bluetooth succeed as a large scale-hoc networking technology?,V 23, issue 3, 644-656.

Wedge, G. (2010), 'Next-Gen Wireless in Control Systems. Wireless as an Enabler - Imagine the Possibilities, 23rd February [Presentation]: IET London: Savoy Place, UK.

Williams, G. (2010), 'Next-Gen Wireless in Control Systems. Wireless Enabled Mobile Solutions', 23rd February [Presentation]: IET London: Savoy Place, UK.

Willig, A. (2003), 'Polling-based MAC protocols for improving realtime performance in a wireless PROFIBUS', Industrial Electronics, IEEE Transactions, 806 - 817.

Willig, A.; Matheus, K. \& Wolisz, A. (2005), 'Wireless Technology in Industrial Networks', Proceedings of the IEEE 93(6), 1130 1151. 